\section{临床试验及相关研究 论文报告规范}

廖庆姣

武汉美捷登生物科技有限公司
很多作者会问我们, 写作还有什么报告规范 吗? 从哪里知道这些规范呢? 因为他们在投稿 Plos $O n e$ 等期刊后, 经常会因为没有提供报告规范的清单 （Checklist）而退稿, 今天我们就来聊聊临床试验及 相关研究报告规范。

临床试验主要服务于人类, 为临床决策和实践 提供重要依据。因此，对临床试验的准确性和有效 性进行评价是十分必要的, 有效的研究报告应该提 供研究潜在偏倚、风险和适用性所需要的重要信息, 而不规范的报告则限制了对研究的准确评价。现在, 为了规范生物医学研究主要是临床研究的学术论文 报道, 国际上一些编辑针对各种研究类型总结统一了 相应的论文报告规范, 都有哪些呢? 下面为您一一 介绍。

1. 随机对照试验报告规范：CONSORT (CONsolidated Standard Of Reporting Trials)，用于规 范报告临床随机对照研究, 现在使用的是 2010 新版, 有 25 条条目, 还有一个流程图, 详细内 容大家可以到其网站 http://www.consort-statement.org/了解。

2. 系统评价和 Meta 分析报告规范 : PRISMA(Preferred Reporting Items for Systematic reviews and Meta-Analysis），用于规范报道随机对照临 床试验的系统评价和 meta 分析, 现在使用的是 2009 版, 是从 QUOROM (QUality Of Reporting Of Meta-analyses) 更新而来, 有 27 条条目, 还有一个 4 步骤的流程图, 详细内容大家可以到 其网站 http://www.prisma-statement.org/了解。

3. 观察性临床实验的 meta 分析报告规范: MOOSE (Meta-analysis Of Observational Studies in

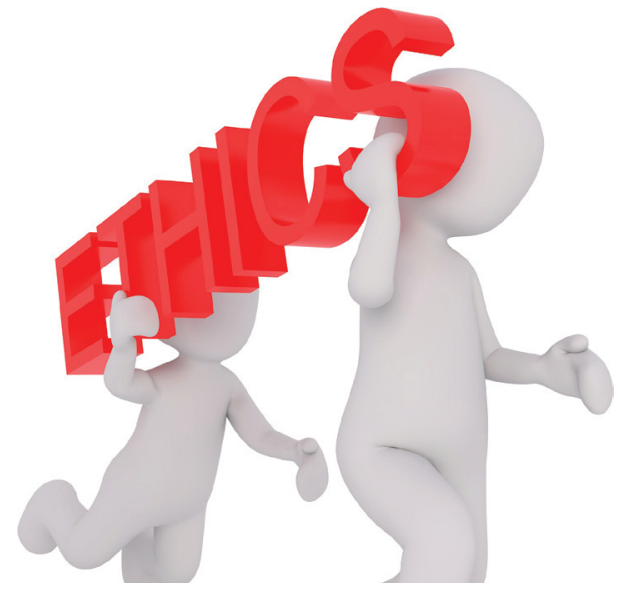

Epidemiology）, 用于报道对观察性研究进行 meta 分析, 有 35 条条目, 这个规范暂时没有网 站，内容首先发表于 JAMA. 2000;283(15):20082012. doi: 10.1001/jama.283.15.2008。有关中文 解读大家可以自行用 moose 规范进行搜索。

4. 非随机对照研究报告规范：TREND (Transparent Reporting of Evaluations with Nonrandomized Designs), 用于规范报道临床非随机对 照研究, 有 22 条条目, 详细内容大家可以到其 网站 http://www.cdc.gov/trendstatement/了解。

5. 观察性研究报告规范：STROBE (STrengthening the Reporting of OBservational studies in Epidemiology), 用于规范报道观察性研究 (包 括队列、病例对照和横断面研究), 有 22 条条目, 2014 年在 Lancet Infectious Diseases 上发表了 STROBE 扩展版本 STROME-ID（STengthening the Reporting of Molecular Epidemiology for Infectious Diseases)，主要是针对感染性疾 病的, 在 STROBE 基础上又加入了 20 条条目, 详细内容大家可以到其网站 http://www.strobestatement.org/解。

6. 基因相关性研究规范报告: STREGA（STrengthening the REporting of Genetic Associations), 用于规范报道基因遗传相关性研究, 从 STROBE扩 展修改而来, 有 22 条条目, 详细内容大家可以到 其网站 http://www.med.uottawa.ca/public-healthgenomics/web/eng/strega.html了解。

7. 诊断试验报告规范: STARD (STAtement for Reporting studies of Diagnostic accuracy), 用 于规范报道诊断学研究, 有 25 条条目, 还有一 
个流程图, 详细内容大家可以到其网站 http:// www.stard-statement.org/了解。

8. Case report 报告规范：CARE（CAse REports), 用于规范报道病例报告, 有 13 条条目, 详细内容 大家可以到其网站 http://www.care-statement.org/ index.html\#contact了解。

9. 动物实验报告规范: ARRIVE (Animal Research: Reporting of In Vivo Experiments), 用于规范报道动物实验, 有 20 条条目, 详细内 容大家可以到其网站 http://www.nc3rs.org.uk/ arrive-guidelines了解。

10. 手术队列研究报告规范 : STROCSS (Strengthening the reporting of cohort studies in surgery), 用于报道手术队列 研究, 2017 年新出的规
范, 共有 17 条条目, 详细内容大家可以到其网站 进行了解 https://www.strocssguideline.com/

11. 肿瘤标志物预后研究报告规范: REMARK (REporting Recommendations for tumour MARKer prognostic studies)用于肿瘤诊断和 预后研究, 观察性研究, 有 20 多条条目, 详 细内容大家可以到其网站进行了解 http://www. equator-network.org/reporting-guidelines/ reporting-recommendations-for-tumour-markerprognostic-studies-remark/。

如果大家想一次了解多种报告规范的要求, 可以 到综合网站 http://www.equator-network.org/进行了 解, 它列出了多种报告规范及其相应的下载链接和要 求。

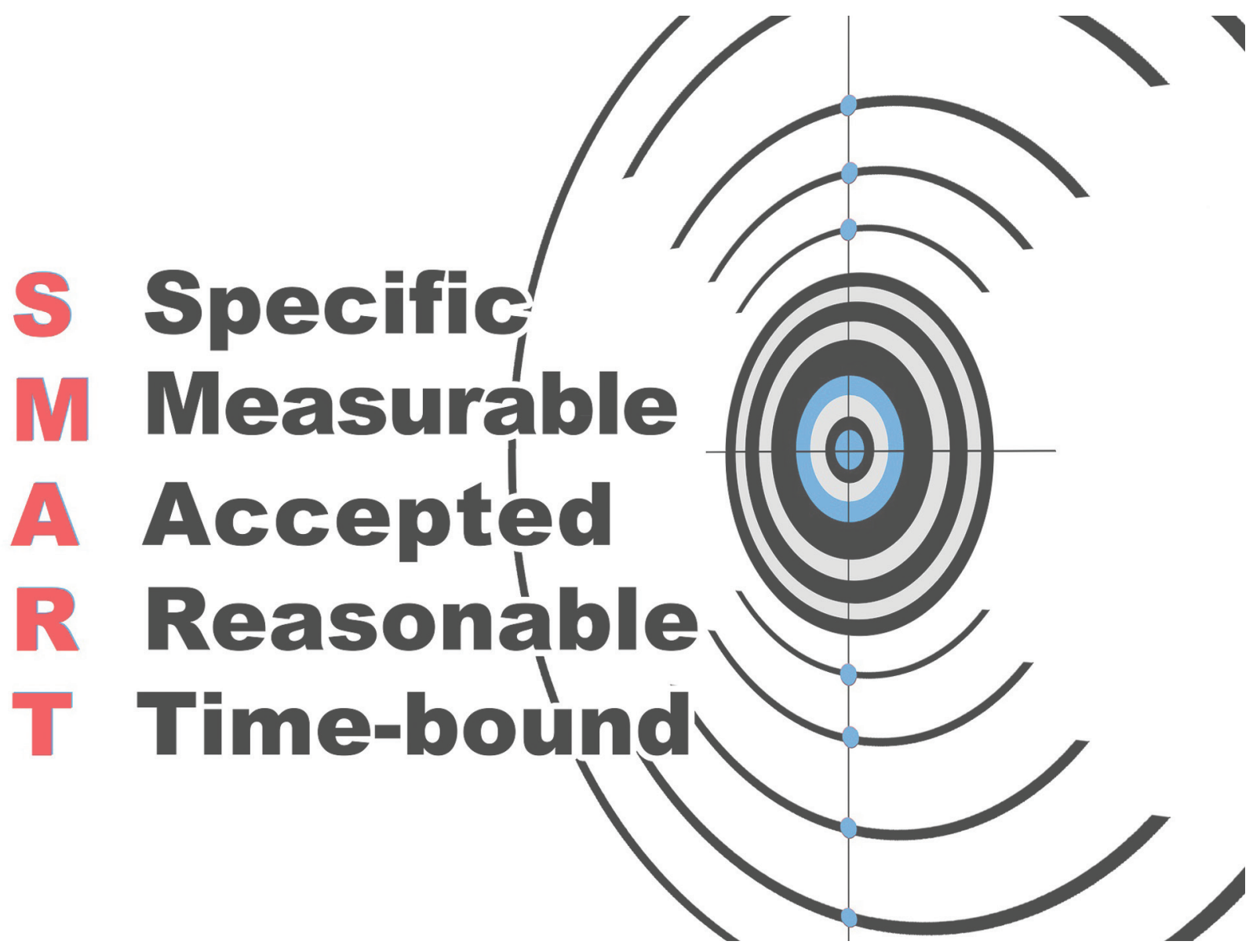

